\title{
Axon Guidance Molecules and Neural Circuit Remodeling After Spinal Cord Injury
}

\author{
Edmund R. Hollis II ${ }^{1,2}$ (i)
}

Published online: 16 December 2015

(C) The American Society for Experimental NeuroTherapeutics, Inc. 2015

\begin{abstract}
...once the development was ended, the founts of growth and regeneration of the axons and dendrites dried up irrevocably.

Santiago Ramón y Cajal
\end{abstract}

Cajal's neurotropic theory postulates that the complexity of the nervous system arises from the collaboration of neurotropic signals from neuronal and non-neuronal cells and that once development has ended, a paucity of neurotropic signals means that the pathways of the central nervous system are "fixed, ended, immutable". While the capacity for regeneration and plasticity of the central nervous system may not be quite as paltry as Cajal proposed, regeneration is severely limited in scope as there is no spontaneous regeneration of long-distance projections in mammals and therefore limited opportunity for functional recovery following spinal cord injury. It is not a far stretch from Cajal to hypothesize that reappropriation of the neurotropic programs of development may be an appropriate strategy for reconstitution of injured circuits. It has become clear, however, that a significant number of the molecular cues governing circuit development become re-active after injury and many assume roles that

Electronic supplementary material The online version of this article (doi:10.1007/s13311-015-0416-0) contains supplementary material, which is available to authorized users.

Edmund R. Hollis, II edh3001@med.cornell.edu

1 Burke Medical Research Institute, White Plains, NY, USA

2 Brain and Mind Research Institute, Weill Cornell Medical College, New York, NY, USA paradoxically obstruct the functional re-wiring of severed neural connections. Therefore, the problem to address is how individual neural circuits respond to specific molecular cues following injury, and what strategies will be necessary for instigating functional repair or remodeling of the injured spinal cord.

Key Words Wnt · semaphorin · ephrin · SCI · axon plasticity $\cdot$ functional recovery

\section{Guidance Molecules Define Connectivity During Development and are Active After Spinal Cord Injury}

The exquisite complexity of connections between the billions of neurons of the human central nervous system (CNS) is orchestrated by a series of molecular cues that dictate the initiation, trajectory, and connectivity of each individual axon. These guidance molecules mediate attraction and repulsion, through direct cellular interactions and long-distance diffusion, all of which results in fasciculation, turning, and eventual target innervation [1]. Expression of these cues changes throughout development as the nervous system begins to take shape. If the failure to regenerate after spinal cord injury (SCI) is due, in part, to the lack of appropriate guidance [2], utilizing the original developmental pathways to mediate reconnectivity is an intellectually appealing approach. This concept is buttressed by the success of fetal neural cell grafts in integrating with the damaged adult CNS. Not only do these developmentally naïve neurons grow axons extensively throughout the adult nervous system, they also provide a growth permissive target for regeneration of injured adult axons [3-5]. This suggests that molecular cues present in the embryonic grafts are sufficient for promoting regeneration of axons in the adult spinal cord. 
An abundance of guidance molecules are now known to mediate neural circuit assembly during development [1]. However, much less is known about the actions of these molecules in the adult CNS. Several members of the canonical guidance molecule families, including netrins, slits, semaphorins, and ephrins, are expressed throughout adulthood in the brain and spinal cord, where their roles in processes such as ongoing neurogenesis and synapse refinement are only beginning to be illuminated [6-10]. Furthermore, members of nearly all classes of guidance molecules yet described have been put forward as responding to or governing aspects of the CNS injury response [10]. As in development, guidance molecules are involved in not only the axonal response, but have also been shown to affect the response of glia, vasculature, and the immune system after injury [10-12]. Netrins and slits have been found to be upregulated following SCI; however, the functional effects of netrin and slit modulation have not yet been demonstrated [13, 14]. Members of the ephrin, semaphorin, and Wnt families govern aspects of ascending sensory and descending motor pathway guidance, are reexpressed after SCI, and have been proposed to inhibit neural circuit plasticity and functional recovery after SCI [10]. While most classes of guidance molecules can act bimodally, mediating attraction or repulsion dependent upon downstream signaling mechanisms, after injury the response of injured axons to ephrins, semaphorins, and Wnts has been largely one of repulsion [10]. Successful regeneration of spinal tracts may require modulation of guidance molecule signaling after injury by either inhibiting the repulsive response or switching it towards attraction. This review will focus on how guidance molecules affect injured spinal cord circuits and the functional consequences of manipulating these signaling pathways.

\section{SCI and Scarring}

The initial result of SCI is the interruption of ascending and descending circuits, the death of proximal neurons and glia, and an immediate loss of function [15]. Following SCI, the lesion is rapidly invaded by neutrophils, macrophages, and microglia $[16,17]$, leading to inflammatory cascades that cause secondary degeneration of glia and axons, cystic cavitation, and the eventual establishment of a glial scar $[16,17]$. The scar is composed of the surrounding reactive astroglial matrix, chondroitin sulfate proteoglycan (CSPG) deposition, oligodendrocyte precursor cells expressing the CSPG NG2, and a fibrotic core $[17,18]$. Astrocytes within the reactive astroglial matrix secrete several growth-restrictive CSPGs $[17,19]$; however, regenerating spinal cord axons are frequently associated with astroglial protrusions within the glial scar [20, 21]. In co-culture experiments, primary sensory neurons will preferentially extend neurites on astrocyte monolayers and actively avoid meningeal fibroblasts [22, 23], suggesting that the fibrotic core of the scar is a greater barrier to regeneration than the astroglial matrix. In vivo, leptomeningeal fibroblast infiltration results in a significant barrier, preventing re-entry of regenerated sensory axons from a grafted scaffold to the host spinal cord [24]. The scar also serves as a source of repulsive guidance molecules, with astrocyte production of ephrinB2 and fibroblast secretion of class 3 semaphorins $[11,25,26]$.

\section{Semaphorins}

Vertebrate semaphorins fall into 5 classes: class 3 semaphorins (Sema3) are secreted and signal through a neuropilin/plexin complex, while classes 4-7 (Sema4-7) are membrane associated and can bind plexin receptors directly, as can secreted Sema3E [27, 28]. Semaphorins generally act as repulsive guidance molecules, though they can also mediate attraction, depending upon the receptor complex activated, which, in turn, can be neuronal subtype specific or timing dependent [29]. In the developing spinal cord, various semaphorins dictate the projections of distinct neural populations. Semaphorins prevent inappropriate monosynaptic connections between primary sensory neurons of the peripheral nervous system (PNS) and motor neurons in the spinal cord [30], and control projections at the CNS-PNS interface (Fig. 1) [27]. Large-diameter proprioceptive neurons that form the fibers of the ascending dorsal funiculus are prevented from aberrantly infiltrating the dorsal horn termination zone of cutaneous afferents by transmembrane semaphorin (Sema6C and Sema6D) signaling through PlexinA1 [31].

The activity of semaphorins in preventing aberrant circuit development through repulsive signaling is of concern as expression of class 3-secreted Semaphorins, including Sema3A, is induced in meningeal fibroblasts after SCI (Fig. 1) $[25,26]$. Throughout development and into adulthood, dorsal root ganglia sensory neurons express the secreted Semaphorin coreceptor neuropilin-1 [32, 33]. As such, both embryonic and adult sensory axons are susceptible to the repulsive actions of soluble Sema3A, which mediates growth cone collapse in vitro $[32,33]$. Furthermore, Sema3A can induce dorsal root ganglia

Fig. 1 Semaphorin (Sema) signaling repels proprioceptive axons during development and after spinal cord injury. (a) During development, proprioceptive, trkC $^{+}$axons expressing PlexinA1 are prevented from prematurely entering the spinal cord through the lateral dorsal root entry zone by expression of Sema6C and Sema6D in the dorsal horn. (b) Later in development, Sema3E-PlexinD1 signaling controls synaptic connectivity, but not motor pool specification, by preventing the aberrant formation of monosynaptic connections with PlexinD1expressing proprioceptive afferents. (c) After spinal cord injury, class 3 semaphorin secretion from meningeal fibroblasts limits the regeneration of ascending proprioceptive axons 
a

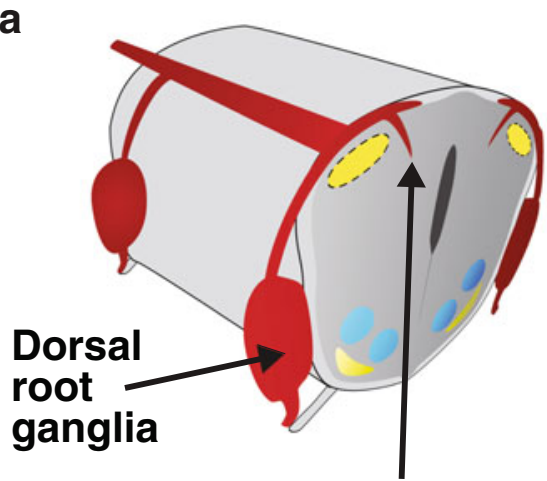

PlexinA1+ proprioceptive afferents avoid dorsolateral Sema6C/6D

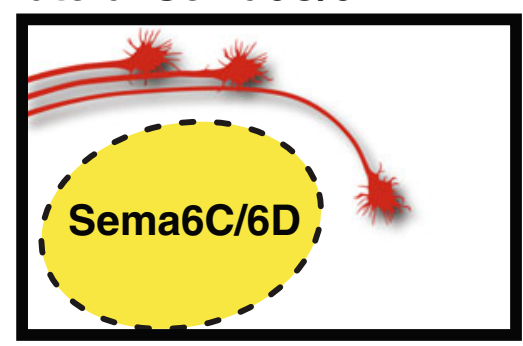

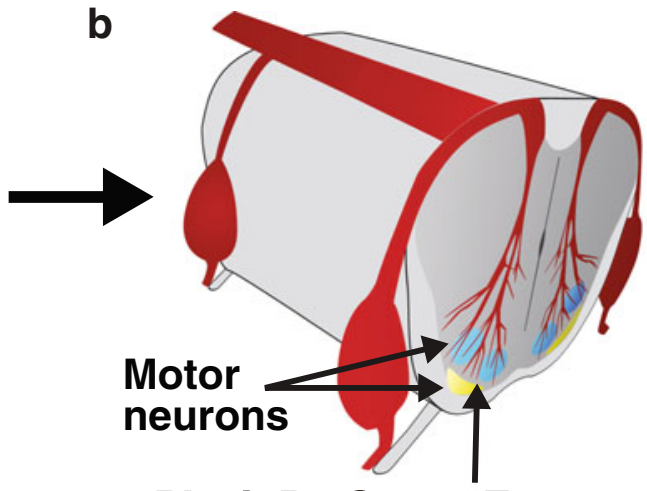

PlexinD1-Sema3E signaling prevents aberrant monosynaptic input to MNs

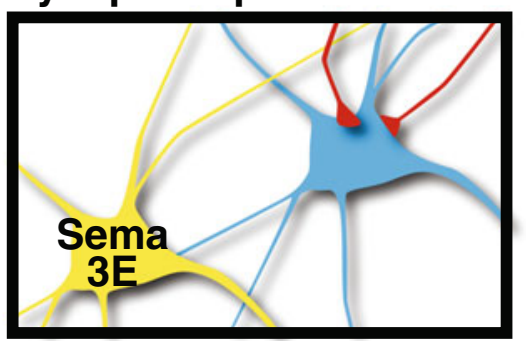

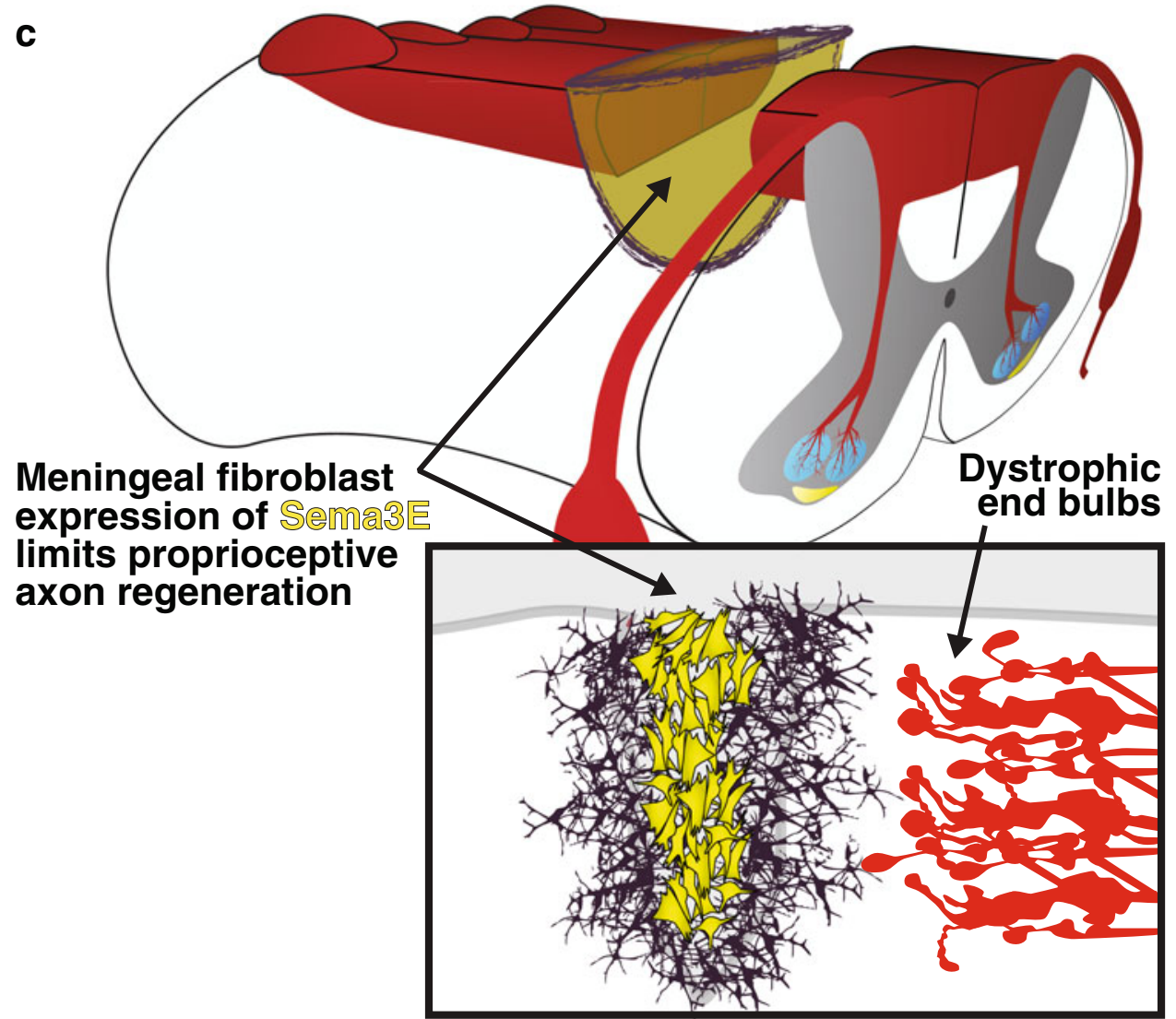


neuron death in vitro, while plexinA3 knockout mice show reduced levels of sensory neuron apoptosis during development $[34,35]$.

In vivo, ascending dorsal column sensory axons that have been conditioned to regenerate in the injured CNS by peripheral nerve injury are unable to bridge regions of Sema3A expression (Fig. 1) [36]. Sema3A also co-localizes with cell surface CSPGs on cortical neurons, increasing the likelihood of Sema3A-neuropilin-1 interaction and axon repulsion [37-39]. This interaction can be augmented by the addition of glycosaminoglycans, which decreases neurite outgrowth and increases growth cone collapse [37, 38]. Conversely, digestion of the chondroitin sulfate side chains with chondroitinase $\mathrm{ABC}$ releases bound biologically active Sema3A, perhaps illuminating a mechanism contributing to the growth-promoting effects of chondroitinase $\mathrm{ABC}$ treatment [28, 37-39].

Two small molecules, SM-216289 and SM-345431, have been generated that inhibit Sema3A binding to neuropilin-1 and block growth cone collapse in vitro [40, 41]. In rodent models of full thoracic spinal cord transection, SM-216289 and SM-345431 promote axonal regeneration into the scar, including regeneration of serotonergic raphaespinal axons [41, 42]. Although there is no clear anatomical evidence that serotonergic axons effectively bridge the lesion site and extend into the caudal stump [41, 42], infusion of the serotonin neurotoxin 5,7-dihydroxytryptamine moderately impairs the SM-216289mediated recovery of slight hindlimb joint movement [42]. Genetic deletion of both signal transducing plexinA 3 and plexinA 4 fails to augment serotonergic axon regeneration [43]. Nevertheless, despite expression of receptors for class 3 semaphorins on intact and injured corticospinal neurons [26], neither small molecule inhibition nor genetic deletion of plexins (A3 and A4) resulted in any demonstrable regeneration of the corticospinal tract, suggesting that other factors may limit corticospinal axon plasticity after injury [41-43].

As described above, inhibition of class 3 semaphorin is associated with slight improvement in locomotor function following complete transection; however, it is unclear whether these effects are due to axon specific signaling and plasticity, axonindependent effects, or some combination thereof [41, 42]. As it does during development, semaphorin signaling may induce neuronal apoptosis after injury, either independent of, or downstream of axonal signaling. In a mouse model of amyotrophic lateral sclerosis, Sema3A is specifically upregulated in terminal Schwann cells at fast-fatigable (subtype $\mathrm{IIb} / \mathrm{x}$ ) neuromuscular junctions, synapses that are susceptible to early loss in the motor neuron disease [44]. The expression pattern of Sema3A may indicate a role in the loss of neuromuscular synapses in amyotrophic lateral sclerosis, an early event in the pathology of the disease, preceding motor neuron loss [45, 46].

In addition to semaphorin signaling in neurons, class 3 semaphorins also exert significant effects on the immune response to injury, remyelination, and revascularization; all potential targets of activity for the small molecule inhibitors described above [12, 41]. Additionally, the small molecule inhibitors of Sema3A have potentially significant off-target effects on ephrins, matrix metalloproteases, and growth factor signaling cascades [41, 42]. Further work will be required to determine whether the functional recovery that follows class 3 semaphorin inhibition is due to circuit remodeling, effects on other aspects of the injury response, or unanticipated off-target effects.

\section{Ephrins}

There are two subclasses of ephrins, glycosylphosphatidylinositol-anchored ephrin As and transmembrane ephrin Bs, which bind the receptor tyrosine kinase Eph receptors [47]. Eph receptors are also split into A and B subclasses that generally exhibit binding to the $A$ and $B$ ephrins, respectively [47]. EphA4, a receptor expressed in corticospinal motor axons during development, can bind both $\mathrm{A}$ and $\mathrm{B}$ subclasses $[47,48]$. Ephrins largely mediate repulsion via signaling through Eph receptor kinase function (forward signaling). Alternatively, repulsion can be mediated via reverse signaling with Eph acting as a ligand to signal through the cytoplasmic tail of an ephrin B or through an ephrin A interaction with a transmembrane protein [49-52]. During development, forward EphA4 signaling prevents midline crossing of ascending primary sensory axons and aberrant re-crossing of corticospinal axons in response to midline ephrinB3 (Fig. 2) [48, 53-55]. After corticospinal development at postnatal day 9 , unilateral motor cortex ablation leads to increased midline crossing of intact corticospinal axons in the spinal cord through the ventral midline, which lacks ephrinB3 expression [56]. This endogenous plasticity allows for limited recovery of motor function and decreases with age as ephrinB3 expression increases throughout the spinal cord [56].

EphA4 is expressed in adult corticospinal motor neurons and accumulates in injured axon tips [56,57]. Knocking out EphA4 has been proposed to promote axon regeneration in a unilateral hemisection model; however, the potential effect of spared axons in that study cannot be discounted [58]. Local sprouting of corticospinal axons appears to occur following infusion of an EphA4 blocking peptide that results in reduced retraction of corticospinal axons from the injury site (Fig. 2) [59]. Perhaps unexpectedly, EphA4 knockdown via intrathecal infusion of an antisense oligodeoxynucleotide increased mechanical allodynia after thoracic contusion injury, though the role of aberrant axonal plasticity in this process was not thoroughly explored [60].

Similar to the effects of blocking EphA4 signaling, ephrinB3 knockout or ephrinB2 conditional knockout in glial fibrillary acid protein immunoreactive astrocytes, results in reduced corticospinal retraction and greater axon sprouting 
(Fig. 2) [61, 62]. EphrinB3 is expressed in oligodendrocytes of the adult spinal cord [63], while ephrinB2 increases in astrocytes following SCI $[11,57]$. Increased recovery of plantar stepping was observed in these studies; however, the developmental effects of ephrinB3 knockout on intraspinal circuitry and gait [53, 54], as well as the potential for contralateral sprouting of intact corticospinal axons after unilateral injury in ephrinB2 conditional knockout mice, may be a confound to interpretation of functional recovery data.

Ephrin signaling may play a role in scar formation following SCI. The astroglial matrix of the scar is reduced in glial fibrillary acid protein-specific ephrinB2 conditional knockout mice, though not in EphA4 knockout mice [61, 64, 65]. EphrinB2 in astrocytes can mediate a bidirectional signal with EphB2 expression in meningeal fibroblasts, shaping the structure of the glial scar [11]. The mechanism for axon inhibition through ephrin signaling is not entirely clear as reverse signaling has been proposed to limit cortical neurite outgrowth in vitro through astrocyte expressed EphA4 [58]. It remains to be seen whether limiting ephrin-mediated repulsive cues results in circuit remodeling as a mechanism to support functional recovery from SCI.

\section{Wnts}

Members of the Wnt glycoprotein family are of particular interest in the study of SCI, owing, in part, to their important role in regulating developmental axon growth along the longitudinal axis of the spinal cord [66]. There are 19 mammalian Wnts with 14 identified receptors, including 10 Frizzled Gprotein coupled receptors and the repulsive related to receptor tyrosine kinase (Ryk) [67]. During development, decreasing gradients of Wnt expression along the rostrocaudal axis mediate the guidance of ascending and descending fiber tracts $[68,69]$. Embryonically, Wnt4 expression in the floor plate attracts postcrossing commissural sensory axons through the Frizzled3 receptor and planar cell polarity signaling pathway $[69,70]$. Perinatally, gradients of Wnt1 and Wnt5a in the dorsal spinal cord drive the caudal growth of corticospinal motor axons through the repulsive Wnt receptor Ryk [68].

The role of the atypical receptor tyrosine kinase Ryk in mediating axon guidance was first discovered in the Drosophila embryo [71]. In flies lacking the Drosophila Ryk homologue, Derailed, neurons that normally extend axons through the anterior commissure show aberrant axonal

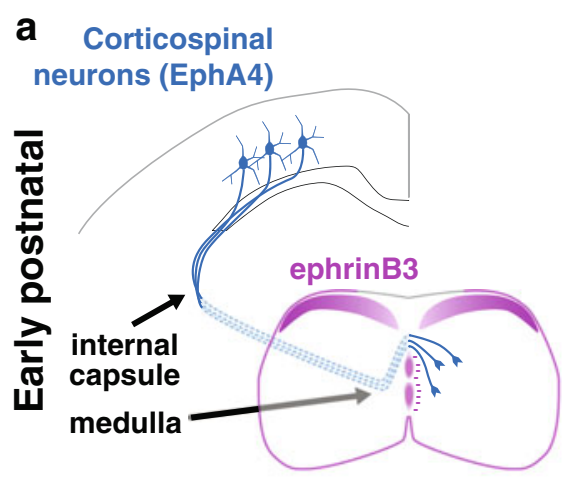

b Corticospinal
neurons (EphA4)

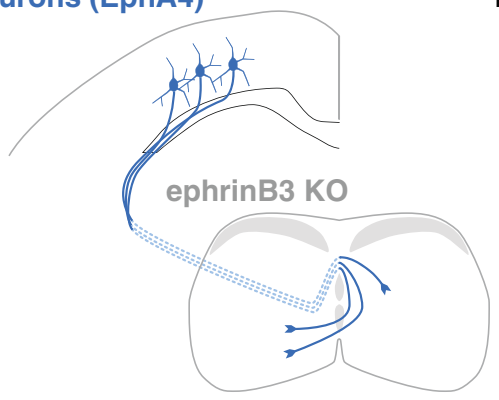

e ephrinB3

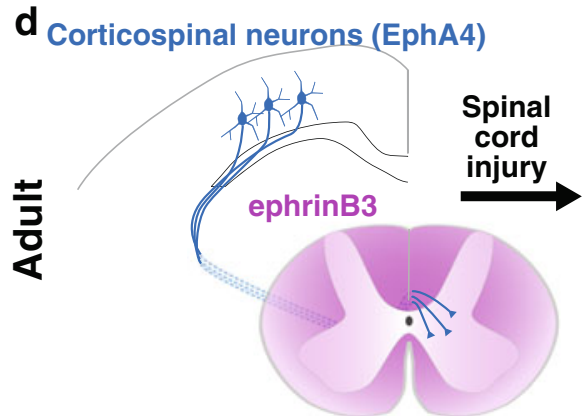

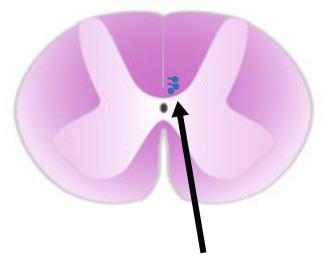

Corticospinal axons (EphA4) f ephrinB3 KO

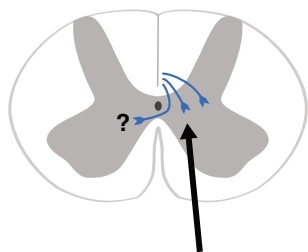

Corticospinal axons (EphA4)

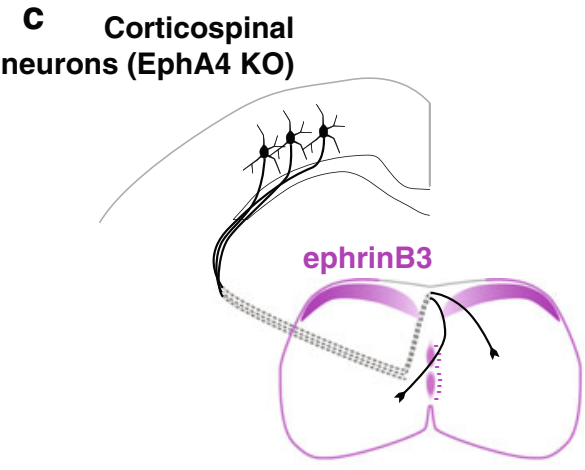

$\mathbf{g}_{\text {ephrinB3 }}$

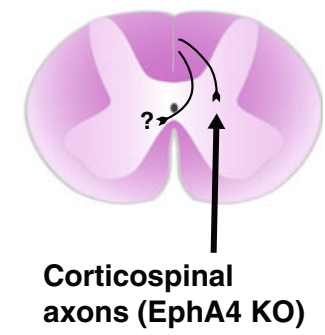

Fig. 2 EphrinB3 repels corticospinal axons through EphA4 forward signaling. (a) Perinatally, ephrinB3 expression (magenta) at the midline prevents re-crossing of EphA4 expressing corticospinal axons (blue) within the spinal cord. (b, c) EphrinB3 and EphA4 knockout mice exhibit re-crossing of corticospinal axons in the spinal cord. EphA4 knockout also results in partial stalling of axons at the medulla and a dorsal shift in the location of descending corticospinal axons (black). (d) In the adult mouse, corticospinal axons express EphA4, and ephrinB3 is expressed by oligodendrocytes in the spinal cord. (e) Following spinal cord injury, ephrinB3-EphA4 signaling limits corticospinal axon plasticity. Mice lacking (f) ephrinB3 or (g) EphA4 show reduced corticospinal axon retraction and greater sprouting after injury. It has not been determined whether ephrinB3-EphA4 deficient corticospinal axon plasticity after injury results in midline crossing (as it does during development) 
projections through the posterior commissure, a region of Wnt5a expression [71, 72]. Conversely, Derailed misexpression in posterior commissure projecting neurons is sufficient to induce repulsion from the posterior commissure [71]. In the mouse, Wnt1 and Wnt5a expression was discovered in the neonatal dorsal spinal cord, in a rostral (high) to caudal (low) gradient [68]. Concurrent with dorsal Wnt gradients, axons of the developing corticospinal tract express Ryk [68]. Attenuation of Wnt-Ryk signaling by polyclonal antibody results in the absence of axon repulsion in cortical explant models in vitro and a stalling of corticospinal axons in vivo [68].

Following development, Wnt family members are either not expressed or are expressed at levels below the threshold for detection in the adult spinal cord. SCI, through either transection or contusion, results in the expression of numerous Wnts surrounding the injury site [73-75]. Wnt expression surrounding the injury site limits axonal plasticity and regeneration of long-distance projections within the dorsal columns $[75,76]$. Additionally, there is evidence that Wnt receptors are expressed locally in neural and non-neuronal cells within the injured spinal cord; however, the role of Wnt signaling on these cells has not yet been defined [77, 78].

Primary sensory neurons within the dorsal root ganglia occupy a unique regenerative niche as they extend axons within both the regeneration-permissive PNS and within the dorsal column of the spinal cord in the nonpermissive CNS.
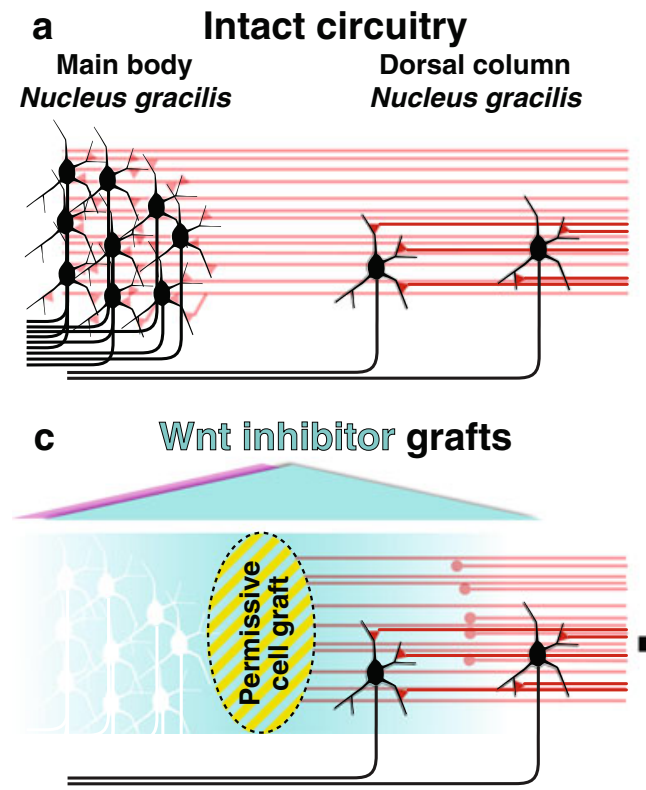

Fig. 3 Wnt inhibition promotes remodeling of spared proprioceptive circuitry and functional recovery. (a) In the intact cervical spinal cord approximately $5 \%$ of second-order dorsal column sensory neurons of nucleus gracilis reside within the cervical spinal cord. These neurons (black) receive mechanoreceptive input from primary sensory axons (red) arising at thoracic level 7 and below. (b) In a cervical level 1 model of spinal cord injury Wnt expression (magenta) is induced surrounding the injury site. Sensory axons that have been previously
Accordingly, neurons of the dorsal root ganglia are capable of peripheral but not central regeneration. However, the growth program that arises following peripheral nerve injury enables regeneration centrally after a subsequent $\mathrm{SCI}$, in a process known as peripheral conditioning [79]. This peripheral conditioning effect has been proposed to be a parallel of the developmental state [80], and indeed results in the reinduction of Ryk expression in mechanoreceptive dorsal root ganglia neurons [76, 81, 82]. While peripheral conditioning allows for regeneration of mechanoreceptive sensory axons after SCI, Ryk expression in these axons sensitizes them to Wnt expression at the spinal cord lesion and limits the amount of induced axon regeneration and plasticity [76]. If Wnt expression is artificially increased after SCI, peripherally conditioned sensory axons are severely repelled and can retract up to several millimeters [76].

Inhibition of Wnt signaling after SCI by cell grafts secreting either of 2 distinct classes of Wnt inhibitor, Wnt inhibitory factor 1 or secreted Frizzled-related peptide 2, enhances regeneration and axon plasticity of ascending dorsal column sensory axons after peripheral conditioning [76]. In a partial injury model at the high cervical level, molecular inhibition of Wnt signaling with secreted Frizzled-related peptide 2 promotes plasticity of the axonal terminals with regeneration into grafted growth-permissive cellular grafts, as well as enhanced synaptic connectivity with spared sensory circuitry in the dorsal column, proximal to the injury site (Fig. 3) [83]. This de

\section{b Injury induced Wnt expression}

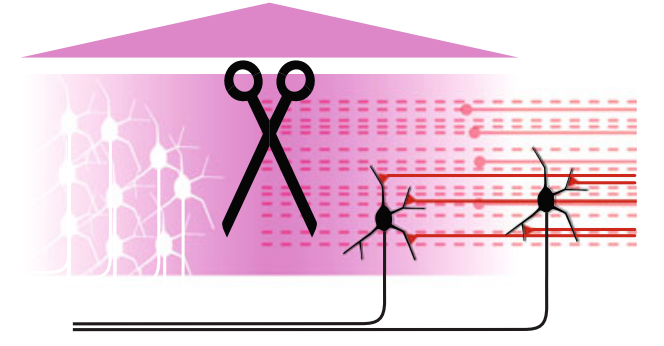

d

Axon plasticity

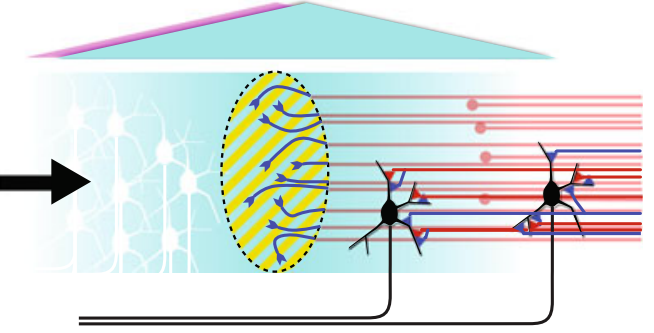

subjected to a peripheral conditioning are sensitive to the re-induced Wnts. (c, d) Bone marrow stromal cell grafts (yellow striped oval) secreting the diffusible Wnt inhibitor SFRP2 (cyan) result in both increased levels of axon regeneration into the grafts (dark blue), as well as increased levels of circuit plasticity proximal to the graft. Synaptic remodeling on spared, nucleus gracilis neurons caudal to the level of injury is sufficient to promote functional recovery 
novo circuit is sufficient for recovery of function on tasks requiring hindlimb proprioceptive input, and is dependent upon the original ascending fibers as transient silencing or transection of the proprioceptive axons below the level of circuit remodeling ablates all functional recovery [83]. In this way, remodeling of the injured sensory circuitry is able to support the recovery of proprioceptive function through a fraction of the remaining original circuitry.

Of the descending motor pathways within the spinal cord, the corticospinal tract is the primary motor circuit for voluntary movement in primates and skilled forelimb movements in rodents [84]. The corticospinal tract has proven reticent to nearly all experimental attempts to promote long-distance regeneration, though it has been shown to be capable of spontaneous short-range sprouting $[85,86]$. However, this sprouting is limited by the re-induction of Ryk expression within the lesioned corticospinal axons [75, 87]. During development, Ryk expressing corticospinal axons encounter a decreasing gradient of Wnts that propels them caudally; meanwhile, injured corticospinal axons face an abrupt barrier of expressed Wnts surrounding the injury site (Fig. 3) $[68,75]$. Blocking of Wnt-Ryk signaling attenuates corticospinal axon retraction from the injury site and allows for greater sprouting of axon collaterals throughout the gray matter [75]. The functional benefit of blocking Wnt-Ryk signaling in corticospinal axons after injury has not yet been demonstrated, though if the enhanced collateralization results in greater connectivity as in the sensory system, it would be expected to enhance the recovery of skilled forelimb usage after SCI.

\section{Conclusions}

The promise of modulating developmental pathways to mediate long-distance regeneration and a reconstitution of injured supraspinal circuitry has yet to be fully realized. In large part this is due to the current limitations in our knowledge of how axon guidance molecules are affecting individual circuits after $\mathrm{SCI}$ and the intrinsic potential of the affected axons to respond appropriately to proregenerative cues. As the effects of guidance molecules after injury so far described are largely inhibiting regeneration, manipulations must be made to block the repulsive activity, switch the response to attraction, or harness the repulsive activity to mediate developmentally appropriate guidance through fasciculation, exclusion, and repulsive propulsion. In addition to direct effects of these powerful molecular cues on neurons, the complexity of the injury response dictates that guidance molecule effects on glia, immune cells, and meningeal fibroblasts must also be considered in attempts to repair damaged spinal cord circuits.

Beyond axon guidance, guidance molecules play other important roles in the development and maintenance of the nervous system, including neurogenesis, polarization, migration, dendrite development, and synapse formation $[7,88,89]$. The guidance molecules reviewed here, ephrins, semaphorins, and Wnts, modulate aspects of synapse formation and stabilization [6-8, 90, 91], critical for circuit remodeling after injury. Recent studies suggest that manipulation of these developmental pathways can induce changes in local connectivity and generate de novo circuits within the injured spinal cord.

While reconstitution of the original circuitry after SCI is an admirable goal, it will require a greater understanding of the spatial and sequential execution of developmental pathways, as well as the complex injury response of individual neural circuits, glia, and mesoderm-derived cells. As animal studies have demonstrated the functional benefits of de novo circuit generation on behavioral recovery from SCI [83, 86, 92, 93], increasing plasticity in zones of partial preservation could yield immediate therapeutic benefits. For example, peripheral nerve transfers allow recovery of arm and hand function in tetraplegic patients through the appropriation of motor units rostral to the injury site $[94,95]$. These proximal motor units, and possibly distal-projecting propriospinal interneurons, constitute legitimate target populations for local corticospinal axon plasticity. Work in both rodent and primate models of SCI indicate that spontaneous local plasticity of the corticospinal motor tract can occur $[85,86,96]$. Additionally, the zone of partial preservation proximal to the injury site is one key neural substrate driving the limited spontaneous recovery that can occur in roughly $40 \%$ of patients after SCI [97]. An understanding of how to enhance local axon plasticity proximal to the injury site and generate appropriate circuit remodeling after injury may prove critical in the recovery from SCI.

Acknowledgments I thank Yimin Zou for postdoctoral support, as well as Corinne Lee-Kubli and Armin Blesch for critical reading of the manuscript. E.R.H. II received financial support from the Craig H. Neilsen Foundation.

\section{Compliance with Ethical standards}

Required Author Forms Disclosure forms provided by the authors are available with the online version of this article.

\section{References}

1. Kolodkin AL, Tessier-Lavigne M. Mechanisms and molecules of neuronal wiring: a primer. Cold Spring Harb Perspect Biol 2011;3: pii.a001727.

2. Ramón y Cajal S. Cajal's degeneration and regeneration of the nervous system. Oxford University Press, New York, 1991.

3. Lu P, Wang Y, Graham L, et al. Long-distance growth and connectivity of neural stem cells after severe spinal cord injury. Cell 2012;150:1264-1273.

4. Lepore AC, Fischer I. Lineage-restricted neural precursors survive, migrate, and differentiate following transplantation into the injured adult spinal cord. Exp Neurol 2005;194:230-242. 
5. Tessler A, Himes BT, Houle J, Reier PJ. Regeneration of adult dorsal root axons into transplants of embryonic spinal cord. J Comp Neurol 1988;270:537-548.

6. Klein R, Kania A. Ephrin signalling in the developing nervous system. Curr Opin Neurobiol 2014;27:16-24.

7. Koropouli E, Kolodkin AL. Semaphorins and the dynamic regulation of synapse assembly, refinement, and function. Curr Opin Neurobiol 2014;27:1-7.

8. Duan Y, Wang S-H, Song J, et al. Semaphorin 5A inhibits synaptogenesis in early postnatal- and adult-born hippocampal dentate granule cells. Elife 2014;3.

9. Jacobi A, Schmalz A, Bareyre FM. Abundant expression of guidance and synaptogenic molecules in the injured spinal cord. PLoS One 2014;9:e88449.

10. Giger RJ, Hollis II ER, Tuszynski MH. Guidance molecules in axon regeneration. Cold Spring Harb Perspect Biol 2010;2.

11. Bundesen LQ, Scheel TA, Bregman BS, Kromer LF. Ephrin-B2 and EphB2 regulation of astrocyte-meningeal fibroblast interactions in response to spinal cord lesions in adult rats. J Neurosci 2003;23: 7789-7800.

12. Mecollari V, Nieuwenhuis B, Verhaagen J. A perspective on the role of class III semaphorin signaling in central nervous system trauma. Front Cell Neurosci 2014;8.

13. Löw K, Culbertson M, Bradke F, Tessier-Lavigne M, Tuszynski MH. Netrin-1 is a novel myelin-associated inhibitor to axon growth. J Neurosci 2008;28:1099-1108.

14. Wehrle R, Camand E, Chedotal A, Sotelo C, Dusart I. Expression of netrin-1, slit-1 and slit-3 but not of slit-2 after cerebellar and spinal cord lesions. Eur J Neurosci 2005;22:2134-2144.

15. Hollis II ER, Tuszynski M. Neurotrophins: Potential therapeutic tools for the treatment of spinal cord injury. Neurotherapeutics 2011;8:694-703.

16. Benowitz LI, Popovich PG. Inflammation and axon regeneration. Curr Opin Neurol 2011;24:577-583.

17. Silver J, Miller JH. Regeneration beyond the glial scar. Nat Rev Neurosci 2004;5:146-156.

18. Jones LL, Yamaguchi Y, Stallcup WB, Tuszynski MH. NG2 is a major chondroitin sulfate proteoglycan produced after spinal cord injury and is expressed by macrophages and oligodendrocyte progenitors. J Neurosci 2002;22:2792-2803.

19. Jones LL, Margolis RU, Tuszynski MH. The chondroitin sulfate proteoglycans neurocan, brevican, phosphacan, and versican are differentially regulated following spinal cord injury. Exp Neurol 2003;182:399-411.

20. Kawaja MD, Gage FH. Reactive astrocytes are substrates for the growth of adult CNS axons in the presence of elevated levels of nerve growth factor. Neuron 1991;7:1019-1030.

21. Liu K, Lu Y, Lee JK, et al. PTEN deletion enhances the regenerative ability of adult corticospinal neurons. Nat Neurosci 2010;13:10751081 .

22. Shearer MC, Niclou SP, Brown D, et al. The astrocyte/meningeal cell interface is a barrier to neurite outgrowth which can be overcome by manipulation of inhibitory molecules or axonal signalling pathways. Mol Cell Neurosci 2003;24:913-925.

23. Shearer MC, Fawcett JW. The astrocyte/meningeal cell interfacea barrier to successful nerve regeneration? Cell Tissue Res 2001;305:267-273.

24. Gros T, Sakamoto JS, Blesch A, Havton LA, Tuszynski MH. Regeneration of long-tract axons through sites of spinal cord injury using templated agarose scaffolds. Biomaterials 2010;31:67196729.

25. Pasterkamp RJ, Giger RJ, Ruitenberg MJ, et al. Expression of the Gene encoding the chemorepellent semaphorin III is induced in the fibroblast component of neural scar tissue formed following injuries of adult but not neonatal CNS. Mol Cell Neurosci 1999;13:143166.
26. De Winter F, Oudega M, Lankhorst AJ, et al. Injury-induced class 3 semaphorin expression in the rat spinal cord. Exp Neurol 2002;175: 61-75.

27. O'Malley AM, Shanley DK, Kelly AT, Barry DS. Towards an understanding of semaphorin signalling in the spinal cord. Gene 2014;553:69-74.

28. Pasterkamp RJ, Verhaagen J. Semaphorins in axon regeneration: developmental guidance molecules gone wrong? Philos Trans R Soc Lond B Biol Sci 2006;361:1499-1511.

29. Koncina E, Roth L, Gonthier B, Bagnard D. Role of semaphorins during axon growth and guidance. Adv Exp Med Biol 2007;621: 50-64.

30. Pecho-Vrieseling E, Sigrist M, Yoshida Y, Jessell TM, Arber S. Specificity of sensory-motor connections encoded by Sema3ePlxnd1 recognition. Nature 2009;459:842-846.

31. Yoshida Y, Han B, Mendelsohn M, Jessell TM. PlexinA1 signaling directs the segregation of proprioceptive sensory axons in the developing spinal cord. Neuron 2006;52:775-788.

32. Kolodkin AL, Levengood DV, Rowe EG, Tai Y-T, Giger RJ, Ginty DD. Neuropilin Is a semaphorin III receptor. Cell 1997;90:753-762.

33. Reza JN, Gavazzi I, Cohen J. Neuropilin-1 is expressed on adult mammalian dorsal root ganglion neurons and mediates semaphorin3a/collapsin-1-induced growth cone collapse by small diameter sensory afferents. Mol Cell Neurosci 1999;14:317-326.

34. Ben-Zvi A, Manor O, Schachner M, Yaron A, Tessier-Lavigne M, Behar O. The semaphorin receptor plexinA3 mediates neuronal apoptosis during dorsal root ganglia development. J Neurosci 2008;28:12427-12432.

35. Ben-Zvi A, Yagil Z, Hagalili Y, Klein H, Lerman O, Behar O. Semaphorin 3A and neurotrophins: a balance between apoptosis and survival signaling in embryonic DRG neurons. J Neurochem 2006;96:585-597.

36. Pasterkamp RJ, Anderson PN, Verhaagen J. Peripheral nerve injury fails to induce growth of lesioned ascending dorsal column axons into spinal cord scar tissue expressing the axon repellent Semaphorin3A. Eur J Neurosci 2001;13:457-471.

37. De Wit J, De Winter F, Klooster J, Verhaagen J. Semaphorin 3A displays a punctate distribution on the surface of neuronal cells and interacts with proteoglycans in the extracellular matrix. Mol Cell Neurosci 2005;29:40-55.

38. Dick G, Tan CL, Alves JN, et al. Semaphorin 3A binds to the perineuronal nets via chondroitin sulfate type $\mathrm{E}$ motifs in rodent brains. J Biol Chem 2013;288:27384-27395.

39. Vo T, Carulli D, Ehlert EME, et al. The chemorepulsive axon guidance protein semaphorin $3 \mathrm{~A}$ is a constituent of perineuronal nets in the adult rodent brain. Mol Cell Neurosci 2013;56:186-200.

40. Kikuchi K, Kishino A, Konishi O, et al. In vitro and in vivo characterization of a novel semaphorin 3A inhibitor, SM-216289 or xanthofulvin. J Biol Chem 2003;278:42985-42991.

41. Zhang L, Kaneko S, Kikuchi K, et al. Rewiring of regenerated axons by combining treadmill training with semaphorin3A inhibition. Mol Brain 2014;7:14.

42. Kaneko S, Iwanami A, Nakamura M, et al. A selective Sema3A inhibitor enhances regenerative responses and functional recovery of the injured spinal cord. Nat Med 2006;12:1380-1389.

43. Lee JK, Chow R, Xie F, Chow SY, Tolentino KE, Zheng B. Combined genetic attenuation of myelin and semaphorinmediated growth inhibition is insufficient to promote serotonergic axon regeneration. J Neurosci 2010;30:10899-10904.

44. Winter FD, Vo T, Stam FJ, et al. The expression of the chemorepellent Semaphorin 3A is selectively induced in terminal Schwann cells of a subset of neuromuscular synapses that display limited anatomical plasticity and enhanced vulnerability in motor neuron disease. Mol Cell Neurosci 2006;32:102-117. 
45. Fischer LR, Culver DG, Tennant P, et al. Amyotrophic lateral sclerosis is a distal axonopathy: Evidence in mice and man. Exp Neurol 2004; 185:232-240.

46. Frey D, Schneider C, Xu L, Borg J, Spooren W, Caroni P. Early and selective loss of neuromuscular synapse subtypes with low sprouting competence in motoneuron diseases. J Neurosci 2000;20:2534-2542.

47. Kullander K, Klein R. Mechanisms and functions of eph and ephrin signalling. Nat Rev Mol Cell Biol 2002;3:475-486.

48. Leighton PA, Mitchell KJ, Goodrich LV, et al. Defining brain wiring patterns and mechanisms through gene trapping in mice. Nature 2001;410:174-179.

49. Holland SJ, Gale NW, Mbamalu G, Yancopoulos GD, Henkemeyer $\mathrm{M}$, Pawson T. Bidirectional signalling through the EPH-family receptor Nuk and its transmembrane ligands. Nature 1996;383:722725 .

50. Brückner K, Pasquale EB, Klein R. Tyrosine phosphorylation of transmembrane ligands for Eph receptors. Science 1997;275:16401643.

51. Bonanomi D, Chivatakarn $\mathrm{O}, \mathrm{Bai} \mathrm{G}$, et al. Ret Is a multifunctional coreceptor that integrates diffusible- and contact-axon guidance signals. Cell 2012;148:568-582.

52. Lim Y-S, McLaughlin T, Sung T-C, Santiago A, Lee K-F, O'Leary DDM. p75NTR mediates ephrin-A reverse signaling required for axon repulsion and mapping. Neuron 2008;59:746-758.

53. Yokoyama N, Romero MI, Cowan CA, et al. Forward signaling mediated by ephrin-B3 prevents contralateral corticospinal axons from recrossing the spinal cord midline. Neuron 2001;29:85-97.

54. Kullander K, Croll SD, Zimmer M, et al. Ephrin-B3 is the midline barrier that prevents corticospinal tract axons from recrossing, allowing for unilateral motor control. Genes Dev 2001;15:877-888.

55. Escalante A, Murillo B, Morenilla-Palao C, Klar A, Herrera E. Zic2-dependent axon midline avoidance controls the formation of major ipsilateral tracts in the CNS. Neuron 2013;80:1392-1406.

56. Omoto S, Ueno M, Mochio S, Yamashita T. Corticospinal tract fibers cross the ephrin-B3-negative part of the midline of the spinal cord after brain injury. Neurosci Res 2011;69:187-195.

57. Fabes J, Anderson P, Yáñez-Muñoz RJ, Thrasher A, Brennan C, Bolsover S. Accumulation of the inhibitory receptor EphA4 may prevent regeneration of corticospinal tract axons following lesion. Eur J Neurosci 2006;23:1721-1730.

58. Goldshmit Y, Galea MP, Wise G, Bartlett PF, Turnley AM. Axonal regeneration and lack of astrocytic gliosis in EphA4-deficient mice. J Neurosci 2004;24:10064-10073.

59. Fabes J, Anderson P, Brennan C, Bolsover S. Regenerationenhancing effects of EphA4 blocking peptide following corticospinal tract injury in adult rat spinal cord. Eur J Neurosci 2007;26:2496-2505.

60. Cruz-Orengo L, Figueroa JD, Velázquez I, et al. Blocking EphA4 upregulation after spinal cord injury results in enhanced chronic pain. Exp Neurol 2006;202:421-433.

61. Ren Z, Chen X, Yang J, et al. Improved axonal regeneration after spinal cord injury in mice with conditional deletion of ephrin B2 under the GFAP promoter. Neuroscience 2013;241:89-99.

62. Duffy P, Wang X, Siegel CS, et al. Myelin-derived ephrinB3 restricts axonal regeneration and recovery after adult CNS injury. Proc Natl Acad Sci U S A 2012;109:5063-5068.

63. Benson MD, Romero MI, Lush ME, Lu QR, Henkemeyer M, Parada LF. Ephrin-B3 is a myelin-based inhibitor of neurite outgrowth. Proc Natl Acad Sci U S A 2005;102:10694-10699.

64. Dixon KJ, Munro KM, Boyd AW, Bartlett PF, Turnley AM. Partial change in EphA4 knockout mouse phenotype: loss of diminished GFAP upregulation following spinal cord injury. Neurosci Lett 2012;525:66-71.
65. Herrmann JE, Shah RR, Chan AF, Zheng B. EphA4 deficient mice maintain astroglial-fibrotic scar formation after spinal cord injury. Exp Neurol 2010;223:582-598

66. Dickson BJ. Wnts send axons up and down the spinal cord. Nat Neurosci 2005;8:1130-1132.

67. Onishi K, Hollis E, Zou Y. Axon guidance and injury-lessons from Wnts and Wnt signaling. Curr Opin Neurobiol 2014;27:232240.

68. Liu Y, Shi J, Lu CC, et al. Ryk-mediated Wnt repulsion regulates posterior-directed growth of corticospinal tract. Nat Neurosci 2005;8:1151-1159.

69. Lyuksyutova AI, Lu C-C, Milanesio N, et al. Anterior-posterior guidance of commissural axons by Wnt-Frizzled signaling. Science 2003;302:1984-1988.

70. Shafer B, Onishi K, Lo C, Colakoglu G, Zou Y. Vangl2 promotes Wnt/planar cell polarity-like signaling by antagonizing Dvl1mediated feedback inhibition in growth cone guidance. Dev Cell 2011;20:177-191.

71. Bonkowsky JL, Yoshikawa S, O'Keefe DD, Scully AL, Thomas JB. Axon routing across the midline controlled by the Drosophila Derailed receptor. Nature 1999;402:540-544.

72. Yoshikawa S, McKinnon RD, Kokel M, Thomas JB. Wnt-mediated axon guidance via the Drosophila Derailed receptor. Nature 2003;422:583-588.

73. Gonzalez-Fernandez C, Fernandez-Martos CM, Shields SD, Arenas E, Javier Rodriguez F. Wnts are expressed in the spinal cord of adult mice and are differentially induced after injury. J Neurotrauma 2014;31:565-581.

74. Fernandez-Martos CM, Gonzalez-Fernandez C, Gonzalez P, Maqueda A, Arenas E, Rodriguez FJ. Differential expression of Wnts after spinal cord contusion injury in adult rats. PLoS One 2011;6:e27000.

75. Liu Y, Wang X, Lu CC, et al. Repulsive Wnt signaling inhibits axon regeneration after CNS injury. J Neurosci 2008;28:8376-8382.

76. Hollis II ER, Zou Y. Reinduced Wnt signaling limits regenerative potential of sensory axons in the spinal cord following conditioning lesion. Proc Natl Acad Sci. 2012;109:14663-14668.

77. Gonzalez P, Fernandez-Martos CM, Arenas E, Rodriguez FJ. The Ryk receptor is expressed in glial and fibronectin-expressing cells after spinal cord injury. J Neurotrauma 2013;30:806-817.

78. Gonzalez P, Fernandez-Martos CM, Gonzalez-Fernandez C, Arenas E, Rodriguez FJ. Spatio-temporal expression pattern of frizzled receptors after contusive spinal cord injury in adult rats. PLoS ONE 2012; 7:e50793.

79. Richardson PM, Issa VM. Peripheral injury enhances central regeneration of primary sensory neurones. Nature 1984;309:791-793.

80. Cai D, Qiu J, Cao Z, McAtee M, Bregman BS, Filbin MT. Neuronal cyclic AMP controls the developmental loss in ability of axons to regenerate. J Neurosci 2001;21:4731-4739.

81. Li X, Li Y-h, Yu S, Liu Y. Upregulation of Ryk expression in rat dorsal root ganglia after peripheral nerve injury. Brain Res Bull 2008;77:178-184.

82. Lu W, Yamamoto V, Ortega B, Baltimore D. Mammalian Ryk is a Wnt coreceptor required for stimulation of neurite outgrowth. Cell 2004; 119:97-108.

83. Hollis II ER, Ishiko N, Pessian M, et al. Remodelling of spared proprioceptive circuit involving a small number of neurons supports functional recovery. Nat Commun 2015;6:6079.

84. Lemon RN, Griffiths J. Comparing the function of the corticospinal system in different species: Organizational differences for motor specialization? Muscle Nerve 2005;32:261-279.

85. Rosenzweig ES, Courtine G, Jindrich DL, et al. Extensive spontaneous plasticity of corticospinal projections after primate spinal cord injury. Nat Neurosci 2010;13:1505-1510. 
86. Ghosh A, Haiss F, Sydekum E, et al. Rewiring of hindlimb corticospinal neurons after spinal cord injury. Nat Neurosci 2010;13:97-104.

87. Miyashita T, Koda M, Kitajo K, et al. Wnt-Ryk signaling mediates axon growth inhibition and limits functional recovery after spinal cord injury. J Neurotrauma 2009;26:955-964.

88. Fenstermaker AG, Prasad AA, Bechara A, et al. Wnt/planar cell polarity signaling controls the anterior-posterior organization of monoaminergic axons in the brainstem. J Neurosci 2010;30: 16053-16064.

89. Salinas PC, Zou Y. Wnt signaling in neural circuit assembly. Annu Rev Neurosci 2008;31:339-358.

90. Dickins EM, Salinas PC. Wnts in action: from synapse formation to synaptic maintenance. Front Cell Neurosci 2013;7:162.

91. Nagaoka T, Ohashi R, Inutsuka A, et al. The Wnt/planar cell polarity pathway component Vangl2 induces synapse formation through direct control of N-cadherin. Cell Reports 2014;6:916-927.
92. Courtine G, Song B, Roy RR, et al. Recovery of supraspinal control of stepping via indirect propriospinal relay connections after spinal cord injury. Nat Med 2008;14:69-74.

93. van den Brand R, Heutschi J, Barraud Q, et al. Restoring voluntary control of locomotion after paralyzing spinal cord injury. Science 2012;336:1182-1185.

94. Brown JM. Nerve transfers in tetraplegia I: Background and technique. Surg Neurol Inte 2011;2:121.

95. Fox I, Davidge K, Novak C, et al. Use of peripheral nerve transfers in tetraplegia: evaluation of feasibility and morbidity. HAND 2015;10:60-67.

96. Bareyre FM, Kerschensteiner M, Raineteau O, Mettenleiter TC, Weinmann O, Schwab ME. The injured spinal cord spontaneously forms a new intraspinal circuit in adult rats. Nat Neurosci 2004;7: 269-277.

97. Fawcett JW, Curt A, Steeves JD, et al. Guidelines for the conduct of clinical trials for spinal cord injury as developed by the ICCP panel: spontaneous recovery after spinal cord injury and statistical power needed for therapeutic clinical trials. Spinal Cord 2006;45:190-205. 